\title{
Inner-outer factorization of operator-valued functions on ordered groups
}

\author{
by \\ Mihály Bakonyi (Atlanta, GA) and Dan Timotin (Bucureşti)
}

\begin{abstract}
Inner-outer factorization for matrix-valued functions defined on totally ordered groups has been considered by Helson and Lowdenslager in connection with multivariate prediction theory. We discuss their result in an operator-theoretic framework and prove that there are obstructions to its extension to operator-valued functions.
\end{abstract}

1. Introduction. Extensions in different directions of the standard inner-outer factorization of analytic functions have been the object of extensive study (see, for instance, [7] and the references therein). We are mainly interested in the setting of totally ordered discrete groups and their duals. This line of generalization already appears in the fundamental paper of Helson and Lowdenslager ([5]), where matrix-valued analytic functions are considered.

We intend to give a more geometric insight into those results. In the classical case, there is an operator-theoretic framework based on dilation theory in Hilbert space, in which the main role is played by the Wold decomposition of an isometry. This line of argument has to be refined in the case of ordered groups, mainly due to the fact that Wold decomposition has to be replaced with other tools. In the scalar-valued case, these ideas have been pursued at length mostly by Helson; a good reference is [4].

For the vector-valued case, an analogue of the theory for semigroups of isometries has been developed; see, for instance, [10, Ch. 9], and the references therein. By using this general theory we are able, firstly, to recapture in this operator framework the results for matrix-valued functions in [5]; secondly, to show how the extension to operator-valued functions faces some basic obstructions.

2. Preliminaries. A basic reference for ordered groups is [8]. Let $G$ be a compact connected abelian group, whose Haar measure will be denoted by $d x$. Its dual $\Gamma$ is a discrete abelian group, and the connectedness of $G$

2000 Mathematics Subject Classification: 47A56, 47A68. 
implies that $\Gamma$ can be totally ordered, not necessarily in a unique manner. We will fix henceforth a total order on $\Gamma$, and will denote by $\Gamma_{+}$the set of nonnegative elements of $\Gamma$. The group operation on $\Gamma$ will be denoted as addition, and, consequently, its unit will also be denoted by 0 . As usual, $C(G)$ will denote the $C^{*}$-algebra of continuous complex-valued functions on $G$.

The Fourier transform $\widehat{f}$ of a function $f \in L^{1}(G)$ is defined by the formula

$$
\widehat{f}(\gamma)=\int_{G} \overline{\gamma(x)} f(x) d x .
$$

One can define equivalents, for $G$, of the Hardy spaces on the unit circle $\mathbb{T}$. We have, for $1 \leq p \leq \infty, H^{p}(G)=\left\{f \in L^{p}(G) \mid \widehat{f}(\gamma)=0\right.$ if $\left.\gamma \notin \Gamma_{+}\right\}$. To pursue further the analogy, one defines inner and outer functions. An inner function $f \in H^{2}(G)$ is characterized, as in the classical case, by the condition $|f|=1$ almost everywhere on $G$. We will call $f \in H^{2}(G)$ an outer function if the closed span of $\gamma f, \gamma \in \Gamma_{+}$, is equal to the whole of $H^{2}(G)$. This is known to be equivalent to the condition that $\widehat{f}(0) \neq 0$ and

$$
\int_{G} \log |f(x)| d x=\log \left|\int_{G} f(x) d x\right| .
$$

In contrast to the case $G=\mathbb{T}$, for a function $f \in H^{2}(G)$ one may have $\int_{G} \log |f(x)| d x=-\infty$. However, if this is not the case, one has a factorization theorem $([8,8.5 .2])$ : for such an $f$ we have $f=f_{\mathrm{i}} f_{\mathrm{o}}$, with $f_{\mathrm{i}}$ inner and $f_{\mathrm{o}}$ outer, and both factors are determined up to a constant of modulus one. Also, an outer function is determined, up to a unitary constant, by its modulus.

We may extend these notions to the case of vector- and operator-valued functions. Namely, if $\mathcal{E}$ is a Hilbert space, then we may define $L^{2}(G, \mathcal{E})$ as the space of measurable functions $f: G \rightarrow \mathcal{E}$ such that $\int_{G}\|f(x)\|^{2} d x<\infty$, and $H^{2}(G, \mathcal{E})$ as the subspace of $L^{2}(G, \mathcal{E})$ characterized by the fact that the scalar function $\langle f(x) \xi, \eta\rangle$ is in $H^{2}(G)$ for all $\xi, \eta \in \mathcal{E}$. We also define $L^{\infty}\left(G, \mathcal{L}\left(\mathcal{E}, \mathcal{E}^{\prime}\right)\right)$ as the space of functions $\phi: G \rightarrow \mathcal{L}\left(\mathcal{E}, \mathcal{E}^{\prime}\right)$ such that $\left\langle\phi(x) \xi, \xi^{\prime}\right\rangle$ is measurable for all $\xi \in \mathcal{E}$ and $\xi^{\prime} \in \mathcal{E}^{\prime}$, and $\|\phi(x)\|$ is essentially bounded. Formula (1) defines also the Fourier coefficients of a vector-valued or operator-valued function. Finally, $H^{\infty}\left(G, \mathcal{L}\left(\mathcal{E}, \mathcal{E}^{\prime}\right)\right)$ is the subspace of $L^{\infty}\left(G, \mathcal{L}\left(\mathcal{E}, \mathcal{E}^{\prime}\right)\right)$ characterized by the fact that the scalar function $\left\langle f(x) \xi, \xi^{\prime}\right\rangle$ is in $H^{\infty}(G)$ for all $\xi \in \mathcal{E}$ and $\xi^{\prime} \in \mathcal{E}^{\prime}$.

Functions $\phi$ in $L^{\infty}\left(G, \mathcal{L}\left(\mathcal{E}, \mathcal{E}^{\prime}\right)\right)$ give rise to multiplication operators from $L^{2}(G, \mathcal{E})$ to $L^{2}\left(G, \mathcal{E}^{\prime}\right)$, which we will denote by $\mathbf{M}_{\phi} . \mathbf{M}_{\phi}$ maps $H^{2}(G, \mathcal{E})$ into $H^{2}\left(G, \mathcal{E}^{\prime}\right)$ if and only if $\phi \in H^{\infty}\left(G, \mathcal{L}\left(\mathcal{E}, \mathcal{E}^{\prime}\right)\right)$; in this case we will denote the corresponding restriction by $\mathbf{T}_{\phi}$. In particular, scalar functions $\phi$ defined 
on $G$ have associated multiplication operators $\mathbf{M}_{\phi}$ and $\mathbf{T}_{\phi}$; it will always be clear from the context on which space the corresponding operators act.

An operator-valued analytic function $\phi \in H^{\infty}\left(G, \mathcal{L}\left(\mathcal{E}, \mathcal{E}^{\prime}\right)\right)$ is called outer if the closed linear span of $\gamma \phi \xi$, for $\gamma \in \Gamma_{+}$and $\xi \in \mathcal{E}$, is the whole $H^{2}\left(G, \mathcal{E}^{\prime}\right)$. It is called inner if $\mathbf{T}_{\phi}$ is an isometry.

The following two lemmas sum up some basic properties that will be used in what follows. Their proofs follow the classical arguments in the case $G=\mathbb{T}$.

Lemma 2.1. (i) An operator $T: H^{2}(G, \mathcal{E}) \rightarrow H^{2}\left(G, \mathcal{E}^{\prime}\right)$ is of the form $\mathbf{T}_{\phi}$ for some function $\phi \in H^{\infty}\left(G, \mathcal{L}\left(\mathcal{E}, \mathcal{E}^{\prime}\right)\right)$ if and only if $T \mathbf{T}_{\gamma}=\mathbf{T}_{\gamma} T$ for all $\gamma \in \Gamma_{+}$(note that the two operators $\mathbf{T}_{\gamma}$ in the last formula act on the different spaces $H^{2}(G, \mathcal{E})$ and $\left.H^{2}\left(G, \mathcal{E}^{\prime}\right)\right)$.

(ii) An operator $T: H^{2}(G, \mathcal{E}) \rightarrow L^{2}\left(G, \mathcal{E}^{\prime}\right)$ is of the form $\mathbf{M}_{\phi} \mid H^{2}(G, \mathcal{E})$ for some function $\phi \in L^{\infty}\left(G, \mathcal{L}\left(\mathcal{E}, \mathcal{E}^{\prime}\right)\right)$ if and only if $T \mathbf{T}_{\gamma}=\mathbf{T}_{\gamma} T$ for all $\gamma \in \Gamma_{+}$.

Lemma 2.2. Suppose $\phi \in L^{\infty}\left(G, \mathcal{L}\left(\mathcal{E}, \mathcal{E}^{\prime}\right)\right)$; then the following are equivalent:

(i) $\mathbf{M}_{\phi}$ is isometric;

(ii) $\mathbf{M}_{\phi} \mid H^{2}(G, \mathcal{E})$ is isometric;

(iii) $\phi(x)$ is almost everywhere isometric.

As a corollary, $\phi \in H^{\infty}\left(G, \mathcal{L}\left(\mathcal{E}, \mathcal{E}^{\prime}\right)\right)$ is inner iff $\phi(x)$ is an isometry a.e.

3. Semigroups of isometries. The well known Wold decomposition for isometries on a Hilbert space (see, for instance, [11, Ch. I]) has some analogues for semigroups of isometries indexed by $\Gamma_{+}$. A few definitions are necessary.

Suppose $\{V(\gamma)\}_{\gamma \in \Gamma_{+}}$is a semigroup of isometries acting on the Hilbert space $\mathcal{H}$. We will call such a semigroup quasiunitary if

$$
\bigvee_{\gamma>\gamma^{\prime}} V(\gamma)^{*} V\left(\gamma^{\prime}\right) \mathcal{H}=\mathcal{H}
$$

The semigroup is called totally nonunitary if $\{V(\gamma) \mid \mathcal{M}\}$ quasiunitary for a doubly invariant subspace $\mathcal{M}$ implies $\mathcal{M}=\{0\}$.

Also, a semigroup is called residual if it is quasiunitary, but has no unitary part (that is, $\{V(\gamma) \mid \mathcal{M}\}$ unitary for a doubly invariant subspace $\mathcal{M}$ implies $\mathcal{M}=\{0\})$.

The following analogue of the Wold decomposition is then valid (see [10, $9.2])$.

THEOREM 3.1. Let $\{V(\gamma)\}_{\gamma \in \Gamma_{+}}$be a semigroup of isometries on $\mathcal{H}$. The space $\mathcal{H}$ admits a unique decomposition of the form 


$$
\mathcal{H}=\mathcal{H}_{\mathrm{u}} \oplus \mathcal{H}_{\mathrm{r}} \oplus \mathcal{H}_{\mathrm{t}}
$$

where $\mathcal{H}_{\mathrm{u}}, \mathcal{H}_{\mathrm{r}}$, and $\mathcal{H}_{\mathrm{t}}$ are reducing subspaces, $\left\{V(\gamma) \mid \mathcal{H}_{\mathrm{u}}\right\}_{\gamma \in \Gamma_{+}}$is unitary, $\left\{V(\gamma) \mid \mathcal{H}_{\mathrm{r}}\right\}_{\gamma \in \Gamma_{+}}$is residual, while $\left\{V(\gamma) \mid \mathcal{H}_{\mathrm{t}}\right\}_{\gamma \in \Gamma_{+}}$is totally nonunitary.

Actually, the proof even identifies the subspaces in decomposition (3) as follows. First,

$$
\mathcal{H}_{\mathrm{u}}=\bigcap_{\gamma \in \Gamma_{+}} V(\gamma) \mathcal{H}
$$

Then, if we define the wandering subspace $\mathfrak{L}$ by

$$
\mathfrak{L}=\mathcal{H} \ominus \bigvee_{\gamma>0} V(\gamma) \mathcal{H}
$$

then all subspaces $V(\gamma) \mathfrak{L}, \gamma \in \Gamma_{+}$, are mutually orthogonal, as well as orthogonal to $\mathcal{H}_{\mathrm{u}}$, and

$$
\mathcal{H}_{\mathrm{t}}=\bigoplus_{\gamma \in \Gamma_{+}} V(\gamma) \mathfrak{L}, \quad \mathcal{H}_{\mathrm{r}}=\mathcal{H} \ominus\left(\mathcal{H}_{\mathrm{u}} \oplus \mathcal{H}_{\mathrm{t}}\right)
$$

We will set, for further use, $\mathcal{H}_{\mathrm{q}}=\mathcal{H}_{\mathrm{u}} \oplus \mathcal{H}_{\mathrm{r}}$; then $\left\{V(\gamma) \mid \mathcal{H}_{\mathrm{q}}\right\}$ is quasiunitary.

We will also note the following analogue, for ordered groups, of the Fourier representation results of $[11$, V.3].

LEMMA 3.2. (i) If $\{V(\gamma)\}_{\gamma \in \Gamma_{+}}$is a totally nonunitary semigroup of contractions in $\mathcal{H}$, and $\mathfrak{L}$ is given by (4), then there exists a unitary map $\Phi: \mathcal{H} \rightarrow H^{2}(\mathfrak{L})$ such that $\Phi V(\gamma) \Phi^{*}$ is multiplication by $\gamma$ on $H^{2}(\mathfrak{L})$.

(ii) Suppose $\left\{V^{\prime}(\gamma)\right\}_{\gamma \in \Gamma_{+}}$is another totally nonunitary semigroup of contractions in $\mathcal{H}^{\prime}$, with corresponding unitary $\Phi^{\prime}: \mathcal{H}^{\prime} \rightarrow H^{2}\left(\mathfrak{L}^{\prime}\right)$. If $Q: \mathcal{H} \rightarrow \mathcal{H}^{\prime}$ is a contraction and $Q V(\gamma)=V^{\prime}(\gamma) Q$ for any $\gamma \in \Gamma_{+}$, then there exists a function $\Theta \in H^{\infty}\left(G, \mathcal{L}\left(\mathfrak{L}, \mathfrak{L}^{\prime}\right)\right)$, with $\|\Theta\| \leq 1$, such that $\Phi^{\prime} Q=\Theta \Phi$.

For any group $G$ the semigroup $\left\{\mathbf{M}_{\gamma}\right\}_{\gamma \in \Gamma_{+}}$is unitary, while $\left\{\mathbf{T}_{\gamma}\right\}_{\gamma \in \Gamma_{+}}$ is totally nonunitary. Also, we may obtain a large class of examples by restricting $\mathbf{M}_{\gamma}$ (or $\mathbf{T}_{\gamma}$ ) to an invariant subspace. The next lemma follows by arguments similar to the case $G=\mathbb{T}$.

Lemma 3.3. Suppose $\mathcal{H} \subset H^{2}(G, \mathcal{E})$ is invariant under all $\mathbf{T}_{\gamma}$ for $\gamma \in \Gamma_{+}$, and define the semigroup of isometries $V(\gamma)=\mathbf{T}_{\gamma} \mid \mathcal{H}$. Then:

(i) $\mathcal{H}_{\mathrm{u}}=\{0\}$;

(ii) there exists an inner function $\phi \in H^{\infty}(\mathfrak{L}, \mathcal{E})$ such that $\mathcal{H}_{\mathrm{t}}=\phi H^{2}(\mathfrak{L})$;

(iii) $\mathcal{H}_{\mathrm{r}}=\{0\}$ if and only if there exists an inner function $\phi \in H^{\infty}(\mathfrak{L}, \mathcal{E})$ such that $\mathcal{H}=\phi H^{2}(\mathfrak{L})$. 
Proof. Since

$$
\bigcap_{\gamma \in \Gamma_{+}} V(\gamma) \mathfrak{H} \subset \bigcap_{\gamma \in \Gamma_{+}} V(\gamma) H^{2}(\mathcal{E})=\{0\},
$$

we have $\mathcal{H}_{\mathrm{u}}=\{0\}, \mathcal{H}=\mathcal{H}_{\mathrm{r}} \oplus \mathcal{H}_{\mathrm{t}}$. Applying Lemma 3.2(i) to $V(\gamma) \mid \mathcal{H}_{\mathrm{t}}$, we obtain a unitary map $\Phi: \mathcal{H}_{\mathrm{t}} \rightarrow H^{2}(\mathfrak{L})$. Consider its inverse $\Phi^{*}$ as taking values in the whole $H^{2}(\mathcal{E})$ instead of $\mathcal{H}_{\mathrm{t}}$; it is an isometry that intertwines multiplications on $H^{2}(\mathfrak{L})$ and $H^{2}(\mathcal{E})$. By Lemma 3.2(ii), it has to be multiplication by a function $\phi \in H^{\infty}(\mathfrak{L}, \mathcal{E})$. That $\phi$ is inner follows from the remarks at the end of Section 1.

Conversely, if there exists an inner function $\phi \in H^{\infty}(\mathfrak{L}, \mathcal{E})$ such that $\mathcal{H}=$ $\phi H^{2}(\mathfrak{L})$, then multiplication by $\phi$ produces a unitary equivalence between the semigroups $V(\gamma)$ and $\mathbf{T}_{\gamma}$; since the latter is totally nonunitary, so is the former.

As noted above, multiplication semigroups on $L^{2}$ and $H^{2}$ provide the standard examples for unitary and totally nonunitary semigroups respectively. These cases are similar to the classical one; it is more interesting to give an example for a residual semigroup. Take $\Gamma=\mathbb{R}$ with the discrete topology; then $G$ is the Bohr compactification of the real line. Consider the invariant subspace $\mathcal{H} \subset H^{2}(G)$ spanned by the characters $\gamma \in \Gamma_{+} \backslash\{0\}$. Then $\left(\mathbf{T}_{\gamma} \mid \mathcal{H}\right)_{\gamma \in \Gamma_{+}}$is residual.

Another example of a residual semigroup (that will play a role in Section 5) is obtained by taking $\Gamma=\mathbb{Z}^{2}$ endowed with the lexicographic order; that is, $\Gamma_{+}=\left\{(m, n) \in \mathbb{Z}^{2}: m>0\right.$ or $m=0$ and $\left.n \geq 0\right\}$. If $\mathcal{H} \subset H^{2}(G)$ is spanned by the characters $(m, n), m>0$, then $\left(\mathbf{T}_{\gamma} \mid \mathcal{H}\right)_{\gamma \in \Gamma_{+}}$is residual.

4. Inner-outer factorization. We have discussed in the preliminaries the inner-outer factorization of a scalar-valued analytic function on $G$. We are now interested in a similar factorization result in case the function is operator-valued. Let us call $\Theta \in H^{\infty}(G, \mathcal{L}(\mathcal{E}, \mathcal{F}))$ factorable if there exist $\Theta_{\mathrm{i}}$ inner and $\Theta_{\mathrm{o}}$ outer such that $\Theta=\Theta_{\mathrm{i}} \Theta_{\mathrm{o}}$.

Define $\mathfrak{H}=\overline{\Theta H^{2}(\mathcal{E})} \subset H^{2}(\mathcal{F})$. Then $\mathfrak{H}$ is invariant with respect to the action of $\mathbf{T}_{\gamma}$ for $\gamma \in \Gamma_{+}$, and we can denote by $V(\gamma)$ the restriction of $\mathbf{T}_{\gamma}$ to $\mathfrak{H}$. According to Lemma $3.3, \mathfrak{H}_{\mathrm{u}}=\{0\}, \mathfrak{H}=\mathfrak{H}_{\mathrm{r}} \oplus \mathfrak{H}_{\mathrm{t}}$, and $\mathfrak{H}_{\mathrm{t}}=\phi H^{2}(\mathfrak{L})$ for some inner function $\phi$ (where, as in (4), $\mathfrak{L}$ is defined by $\left.\mathfrak{L}=\mathfrak{H} \ominus \bigvee_{\gamma>0} V(\gamma) \mathfrak{H}\right)$.

The following simple lemma yields a geometric characterization of factorization.

Lemma 4.1. With the above notations, $\Theta$ is factorable iff $\mathfrak{H}_{\mathrm{r}}=\{0\}$.

Proof. If $\mathfrak{H}_{\mathrm{r}}=\{0\}$, take $\Theta_{\mathrm{i}}=\phi$. For any polynomial $p \in H^{2}(\mathcal{E})$, $\Theta p \in \mathfrak{H}$, and therefore $\Theta p=\Theta_{\mathrm{i}} \widetilde{p}$ for some $\widetilde{p} \in H^{2}(\mathfrak{L})$. Moreover, we 
have $\|\widetilde{p}\|=\|\Theta p\| \leq\|\Theta\| \cdot\|p\|$, and the map $p \mapsto \widetilde{p}$ satisfies the hypothesis of Lemma 3.2(ii). It is therefore multiplication by a bounded function $\Theta_{\mathrm{o}} \in H^{\infty}(\mathcal{E}, \mathfrak{L})$. Since the span of $\Theta_{\mathrm{o}} H^{2}(\mathcal{E})$ is the whole of $H^{2}(\mathfrak{L})$, it follows that $\Theta_{\mathrm{o}}$ is outer as required, and we have $\Theta=\Theta_{i} \Theta_{\mathrm{o}}$.

Conversely, if $\Theta$ is factorable, then Lemma 3.3(iii) implies $\mathfrak{H}_{\mathrm{r}}=\{0\}$.

We will now obtain a positive result for factorization in the finite-dimensional case, giving a different proof of a result of Helson-Lowdenslager ([5]). It involves neither the machinery of prediction theory, nor the use of the determinant function, and the arguments are natural in the operator framework.

Proposition 4.2. If $\mathcal{E}$ is finite-dimensional, and $\widehat{\Theta}(0)$ is invertible, then $\Theta$ is factorable.

Proof. Since $\widehat{\Theta}(0)$ is invertible, we have $\operatorname{dim} \mathcal{E}=\operatorname{dim} \mathcal{F}$; we may therefore assume in the rest of the proof that actually $\mathcal{E}=\mathcal{F}$.

According to Lemma 4.1, we have to consider the space $\mathfrak{H}=\overline{\Theta H^{2}(\mathcal{E})} \subset$ $H^{2}(\mathcal{E})$, the restriction $V(\gamma)$ to $\mathfrak{H}$ of the action of $\mathbf{T}_{\gamma}$ for $\gamma \in \Gamma_{+}$, and to show that $\mathfrak{H}_{\mathrm{r}}=\{0\}$.

For every $\xi \in \mathcal{E}$ denote by $\widehat{\xi}$ the orthogonal projection of the constant function $\xi \in H^{2}(\mathcal{E})$ onto $\mathfrak{H}$. Since $\xi \perp \bigvee_{\gamma>0} V(\gamma) \mathfrak{H}$, it follows that $\widehat{\xi} \in \mathfrak{L}$. Moreover, if $\widehat{\xi}=0$, then $\xi \perp \mathfrak{H}$, which would imply $\xi \perp \widehat{\Theta}(0) \mathcal{E}$; since $\widehat{\Theta}(0)$ is invertible, we would have $\xi=0$.

Thus the map $\xi \mapsto \widehat{\xi}$ is one-to-one from $\mathcal{E}$ into $\mathfrak{L}$. We want to show that actually $\mathfrak{L}=P_{\mathfrak{H}} \mathcal{E}$. Consider $f \in \mathfrak{L}, f \perp \mathcal{E}$. We have $f=\lim \Theta p_{n}$, where the limit is in $H^{2}(\mathcal{E})$, and $p_{n}$ are finite sums of characters multiplied by vectors in $\mathcal{E}$. If $p_{n}=x_{n}+p_{n}^{0}$ with $x_{n} \in \mathcal{E}$ and $p_{n}^{0}$ containing only strictly positive characters, then since $f \perp \Theta \gamma H^{2}(\mathcal{E})$ for all $\gamma>0$, it follows that $f=\lim \Theta x_{n}$. Take $\xi \in \mathcal{E}$; then $f \perp \mathcal{E}$ implies

$$
0=\langle\xi, f\rangle=\lim \left\langle\xi, \Theta x_{n}\right\rangle=\lim \left\langle\xi, \widehat{\Theta}(0) x_{n}\right\rangle=\lim \left\langle\widehat{\Theta}(0)^{*} \xi, x_{n}\right\rangle .
$$

Since $\widehat{\Theta}(0)^{*}$ is invertible, it follows that $x_{n}$ is weakly (and thus strongly, $\mathcal{E}$ being finite-dimensional) convergent to 0 . Therefore $f=0$, and $\mathfrak{L}=P_{\mathfrak{H}} \mathcal{E}$; in particular, $\mathfrak{L}$ and $\mathcal{E}$ have the same (finite) dimension.

Consider then $g \in \mathfrak{H}_{\mathrm{r}}=\mathfrak{H} \ominus \mathfrak{H}_{\mathrm{t}}$, and fix $\xi \in \mathcal{E}$. First, as $g \perp \gamma \widehat{\xi}$ for all $\gamma \in \Gamma_{+}$, we have

$$
\int_{G} \gamma(x)\langle\widehat{\xi}(x), g(x)\rangle d x=0, \quad \gamma \in \Gamma_{+} .
$$

But, for $\gamma>0, \gamma g \in \gamma \mathfrak{H}$ and $\widehat{\xi} \in \mathfrak{L}$ imply $\gamma g \perp \widehat{\xi}$, so that

$$
\int_{G} \gamma(x)\langle g(x), \widehat{\xi}(x)\rangle d x=0, \quad \gamma>0 .
$$


Together, (6) and (7) imply that

$$
g(x) \perp \widehat{\xi}(x) \quad \text { a.e. }
$$

Since $\mathfrak{L}=P_{\mathcal{H}} \mathcal{E}$, it follows that $g(x) \perp h(x)$ for any $h \in \mathfrak{L}$. Now, the elements of $\mathfrak{L}$ are $\phi \xi$, with $\xi \in \mathcal{E}$; therefore

$$
0=\langle g(x), \phi(x) \xi\rangle=\left\langle\phi(x)^{*} g(x), \xi\right\rangle .
$$

Thus $\phi(x)^{*} g(x)=0$ a.e. But, since $\mathfrak{L}$ and $\mathcal{E}$ have the same finite dimension, it follows that $\phi(x)$ is almost everywhere a unitary. The same is true about $\phi(x)^{*}$; thus $g=0$ and $\mathfrak{H}_{\mathrm{r}}=\{0\}$ as desired.

It can easily be seen that the condition on $\widehat{\Theta}(0)$ in the statement of Proposition 4.2 can be replaced by the requirement that there exists a first nonzero Fourier coefficient which is invertible. It is well known (see [5] or [8]) that this type of condition is essential to ensure factorability.

5. An example. We intend now to show by an example that Proposition 4.2 is not true if we drop the finite-dimensionality requirement on $\mathcal{E}$. Take $\Gamma=\mathbb{Z}^{2}$, endowed with the lexicographic order; accordingly, we will have $G=\mathbb{T}^{2}$, with the two variables denoted $(v, w)$. Also, for further use, the lower index $v$ or $w$ attached to a function space in one variable indicates the corresponding variable.

It should be noted that the lexicographic group has received some attention recently, in connection with dilation theory and $C^{*}$-algebras ([1], [6]). It is a typical example of a nonarchimedean ordered group.

The construction will use the characteristic function of a contraction; we include the basic facts and refer for details to [11]. The defect operator and defect space of the contraction $T \in \mathcal{L}(H)$ are defined by

$$
D_{T}=\left(I-T^{*} T\right)^{1 / 2}, \quad \mathcal{D}_{T}=\overline{D_{T} H} ;
$$

applying this to $T^{*}$, we obtain $D_{T^{*}}$ and $\mathcal{D}_{T^{*}}$. The characteristic function $\theta$ of the completely nonunitary contraction $T \in \mathcal{L}(H)$ is the analytic function $\theta: \mathbb{D} \rightarrow \mathcal{L}\left(\mathcal{D}_{T}, \mathcal{D}_{T^{*}}\right)$ defined by

$$
\theta(z)=-T^{*}+z D_{T^{*}}(I-z T)^{-1} D_{T} \mid \mathcal{D}_{T} .
$$

It is defined as an analytic function on the disc $\mathbb{D}$, and can be shown to have radial limits almost everywhere on $\mathbb{T}$.

We come now to the operator that we will consider. Take $T \in \mathcal{L}\left(\ell^{2}\right)$ to be $T=\frac{1}{2} S$, where $S$ is the unilateral shift. We have $\mathcal{D}_{T}=\mathcal{D}_{T^{*}}=\ell^{2}$. Since $T^{n} \rightarrow 0$ and $T^{* n} \rightarrow 0$, the general theory of [11] says that its characteristic function defined by (8) is inner and *-inner, whence its boundary values are a.e. unitary operators (this can also be checked directly). Also, $\theta(0)=-\frac{1}{2} S^{*}$ has the kernel $\mathbb{C}_{0}$ of dimension 1 (if $\left(e_{n}\right)$ is the standard orthonormal basis). 
Consider now the function $\theta_{1}(z)=\theta(z) S$. Its main properties are:

- $\theta_{1}(z)$ is an isometry a.e. on $\mathbb{T}$;

- the orthogonal complement of the range of $\theta_{1}(z)$ has dimension 1 , being spanned by the analytic function $\theta(z) e_{0}$;

$-\theta_{1}(0)$ is invertible.

Now, the analytic inner function $\widetilde{\theta}_{1}(z)=\theta_{1}(\bar{z})^{*}$ has the dual properties: its values are almost everywhere coisometries with kernels of dimension 1 , the kernels vary analytically in $z$, and again the value at 0 is invertible. Finally, we define $\Psi$ to be the infinite direct sum of $\theta_{1}$ and $\widetilde{\theta}_{1}$. As a consequence, we obtain the following objects:

(i) an analytic, partial-isometric-valued function $\Psi$, with values in a separable Hilbert space $\mathcal{E}$, with $\Psi(0)$ invertible;

(ii) a sequence of analytic functions $\kappa_{n}: \mathbb{T} \rightarrow \mathcal{E}$, forming, for each $z$, an orthonormal system in the kernel of $\Psi(z)$;

(iii) an analytic function $\psi: \mathbb{T} \rightarrow \mathcal{E}$, with values vectors of norm 1 , such that $\psi(z) \perp \Psi(z) \mathcal{E}$ for each $z$.

Based on these objects, as well as on a sequence of positive numbers $\alpha_{n}$, $n \geq 1$, such that $\sum_{n \geq 1} \alpha_{n}<\infty$, we define the desired function $\Theta: G \rightarrow \mathcal{L}(\mathcal{E})$ by

$$
\Theta(v, w)(\xi)=\Psi(w) \xi+v \sum_{n \geq 1} \alpha_{n} w^{-n}\left\langle\xi, \kappa_{n}(w)\right\rangle \psi(w) .
$$

This is a well defined function in $H^{2}(G, \mathcal{E})$. Indeed, for the first term there is no problem, while all the terms in the convergent series on the right belong to $v H_{v}^{2} \otimes L_{w}^{2} \subset H^{2}(G, \mathcal{E})$. Also, $\Theta(0,0)=\Psi(0)$, and is thus invertible.

We are now interested in $\mathcal{H}=\overline{\Theta H^{2}(G, \mathcal{E})} \subset H^{2}(G, \mathcal{E})$. According to Lemma 4.1, in order to show that $\Theta$ is not factorable, we must prove that $\mathcal{H}_{\mathrm{r}} \neq\{0\}$.

First, note that, for $l \geq 0$ and $n \in \mathbb{Z}$,

$$
\Theta(v, w) v^{l} \kappa_{n}=v^{l+1} w^{-n} \alpha_{n} \psi(w),
$$

and thus $\mathcal{H}_{0}=\left(v H_{v}^{2} \otimes L_{w}^{2}\right) \psi(w) \subset \mathcal{H}$. On the other hand,

$$
\mathcal{H} \subset\left(\Psi(w) H^{2}(G, \mathcal{E})\right) \oplus \mathcal{H}_{0} .
$$

Now, a general Hilbert space argument says that if $\mathcal{H} \subset \mathcal{K} \oplus \mathcal{H}_{0}$, and $\mathcal{H} \supset \mathcal{H}_{0}$, then $\mathcal{H}=\mathcal{H}^{\prime} \oplus \mathcal{H}_{0}$, where $\mathcal{H}^{\prime}=\mathcal{H} \ominus \mathcal{H}_{0} \subset \mathcal{K}$. Moreover, if $\mathcal{H}, \mathcal{K}, \mathcal{H}_{0}$ are invariant under an operator $V$, then $\mathcal{H}^{\prime}$ is also invariant under $V$, and thus $\mathcal{H}^{\prime}, \mathcal{H}_{0}$ are actually reducing.

If we consider $\mathcal{K}=\Psi(w) H^{2}(G, \mathcal{E})$, and instead of $V$ the semigroup of isometries $\{V(\gamma)\}$, it follows that

$$
\mathcal{H}=\mathcal{H}^{\prime} \oplus\left(v H_{v}^{2} \otimes L_{w}^{2}\right) \psi(w)
$$


and thus $\mathcal{H}_{0}=\left(v H_{v}^{2} \otimes L_{w}^{2}\right) \psi(w)$ is a reducing subspace for the semigroup $\{V(\gamma)\}_{\gamma \in \Gamma_{+}}$. Since, moreover,

$$
\left(v H_{v}^{2} \otimes L_{w}^{2}\right) \psi(w)=V((0,1))\left[\left(v H_{v}^{2} \otimes L_{w}^{2}\right) \psi(w)\right],
$$

it follows that

$$
\mathfrak{L}=\mathcal{H}_{0} \ominus \bigvee_{\gamma>0} V(\gamma) \mathcal{H}=\{0\}
$$

and thus, by $(5), \mathcal{H}_{0, \mathrm{t}}=\{0\}$. Consequently, $\left(v H_{v}^{2} \otimes L_{w}^{2}\right) \psi(w)$ is in the residual part of the semigroup $\{V(\gamma)\}_{\gamma \in \Gamma_{+}}$, which is what we intended to prove. Thus $\mathcal{H}_{\mathrm{r}} \neq\{0\}$, whence $\Theta$ is not factorable.

Acknowledgements. This work has been supported by NSF grant INT-0307395. The second author also acknowledges support from CNCSIS grant no. 1620.

\section{References}

[1] R. Bruzual and M. Dominguez, Equivalence between the dilation and lifting properties of an ordered group through multiplicative families of isometries. A version of the commutant lifting theorem on some lexicographic groups, Integral Equations Operator Theory 40 (2001), 1-15.

[2] R. G. Douglas, On factoring positive operator functions, J. Math. Mech. 16 (1966), 119-126.

[3] H. Helson, Lectures on Invariant Subspaces, Academic Press, New York, 1964.

[4] - Analyticity on compact abelian groups, in: Algebras in Analysis (Birmingham, 1973), Academic Press, London, 1975, 1-62.

[5] H. Helson and D. Lowdenslager, Prediction theory and Fourier series in several variables I, Acta Math. 99 (1958), 165-202.

[6] G. J. Murphy, $C^{*}$-algebras generated by commuting isometries, Rocky Mountain J. Math. 26 (1996), 237-267.

[7] M. Rosenblum and J. Rovnyak, Hardy Classes and Operator Theory, Oxford Sci. Publ., Clarendon Press, Oxford Univ. Press, New York, 1985.

[8] W. Rudin, Fourier Analysis on Groups, Interscience, 1962.

[9] D. Sarason, Generalized interpolation in $H^{\infty}$, Trans. Amer. Math. Soc. 127 (1967), 179-203.

[10] I. Suciu, Function Algebras, Editura Academiei, Bucharest, 1973.

[11] B. Sz.-Nagy and C. Foiaş, Harmonic Analysis of Operators on Hilbert Space, American Elsevier, New York, 1970.

Department of Mathematics

Georgia State University

Atlanta, GA 30303, U.S.A.

E-mail: mbakonyi@mathstat.gsu.edu
Institute of Mathematics of the Romanian Academy PO Box 1-764 Bucureşti 014700, Romania E-mail: Dan.Timotin@imar.ro

Received August 2, 2004

Revised version March 2, 2005 